\title{
Significant relationship between parental and child dental fear
}

\author{
Abstracted from \\ Themessl-Huber M, Freeman R, Humphris G, MacGillivary S and Terzi N. \\ Empirical evidence of the relationship between parental and child dental fear: \\ a structured review and meta-analysis. Int J Paediatr Dent 2010; 20: 83-101 \\ Address for correspondence: Markus Themessl-Huber, Department of Behavioural and Social Sciences, \\ CQUniversity, Bruce Highway, Rockhampton, 4701 Q'ld, Australia. E-mail: m.themessl-huber@cqu.edu.au
}

\section{Questions: What is the link between parental and child dental fear?}

Data sources The databases CINAHL,EMbase, Medline, PsycINFO, Cochrane/DARE EBM were searched using OVID together with the reference lists of retrieved papers.

Study selection Studies in English and German which investigated the relationship between parental and child dental fear and included children and young people aged 0-19 years were included. Particular emphasis was placed on studies which used established behaviour and anxiety measures to assess parental and child dental anxiety or child behaviour.

Data extraction and synthesis A qualitative synthesis of the included studies was conducted with those studies which provided sufficient quantitative estimates of association between parental and child measures to enable transformation into effect sizes being used in a meta-analysis. A random-effects model was used.

Results Forty-three studies were included from across the six continents. The studies ranged widely with respect to research design, methods used, age of children included and the reported link between parental and child dental fear. The majority of studies confirmed a relationship between parental and child dental fear. This relationship is most evident in children aged 8 years and under. A meta-analysis confirmed an association between parental and child dental fear. Conclusions The narrative synthesis as well as the meta-analysis demonstrate a significant relationship between parental and child dental fear, particularly in children aged 8 years and under.

\section{Commentary}

This review is well justified by the $10 \%$ mean prevalence of child dental fear which presents a considerable challenge not only to the wellbeing of the children but also to their parents and oral health personnel. Themessl-Huber et al. have made a considerable effort in this structured literature review and meta-analysis to assess the conflicting evidence on the link between parental and child dental fear. This link is supported by theory, but it is also important for the understanding of practitioners, in order to prevent and treat dental fear among children.

As the authors point out, their approach to the literature was not systematic but structured. Also, since 2007, new evidence on the link between parental and child dental fear has been published. ${ }^{1-3}$ The role and importance of using established and validated measures is well documented and a clear shortcoming in many studies. The authors have also made a good attempt to structure the analysis based on the child's age, a very important but often neglected issue, even though the study designs made this attempt rather challenging.

However, the role of the informants in the designs of the selected studies could also have been considered. Recent studies ${ }^{2,3}$ have pointed out that the reported association between family members' dental fear, especially among younger children, is often based on designs where the parent evaluated both his/her and the child's dental fear. This might have lead to a bias and partly explain the controversy in the results.

The authors also suggest that the dental setting might affect the relationship between parental and child dental fear, for example by influencing the likelihood of presenting dental fear. Another possibility is that parents having dental fear are more likely to search for a certain type of care in order to prevent their children from further fear. On the other hand, results among the general population, not selected by attendance or dental setting, suggest a rather weak relationship between the fear of children and their parents. ${ }^{1,2}$ This is also suggested to be partly due to the fact that they do not know about each other's fear. ${ }^{2,3}$

Themessl-Huber et al. are to be congratulated for their suggestions for further research. A more thorough look at the child-patient dyad as well as the role of fathers, almost neglected in previous studies, is also needed, not forgetting factors behind the well established gender difference in the prevalence of dental fear.

Satu Lahti and Anni Luoto Department of Community Dentistry, University of Oulu, Oulu, Finland.

1. Rantavuori K, Lahti S, Tolvanen M, Seppä L, Hausen H. Factors associated with different measures of dental fear among children at different ages. J Dent Child 2009; 76: 13-19.

2. A, Arnrup K, Broberg A, Bodin L, Berggren U. Child dental fear as measured with the children's fear survey schedule: the impact of referral and type of informant (child versus parent). Community Dent Oral Epidemiol 2010; published online: doi:10.1111/ j.1600-0528.2009.00521.x

3. Luoto A, Tolvanen M, Rantavuori K, Pohjola V, Lahti S. Can parents and children evaluate each other's dental fear? Eur J Oral Sci 2010; 118: 254-258. 